\title{
Redirected Cytolysis
}

National Cancer Institute

\section{Source}

National Cancer Institute. Redirected Cytolysis. NCI Thesaurus. Code C15851.

The killing of target cells by changing the natural specificity of cytotoxic T cells by the use of an antibody or other molecule capable of binding to both the $T$ cell and to the target cell. Often involves hybrid (bispecific) antibodies having distinct specificities for the T cell and for the targ et cell. (NCl/OSP) 